\title{
Comparative study of ultrasonic elastography and conventional ultrasound in diagnosis of malignant anus neoplasm
}

\author{
YICHENG ZHU, SHUHAO DENG, YUAN ZHANG and QUAN JIANG \\ Department of Ultrasound, Shanghai Pudong New Area People's Hospital, Shanghai 201299, P.R. China
}

Received November 16, 2017; Accepted December 12, 2017

DOI: $10.3892 /$ etm.2018.5720

\begin{abstract}
The application value of conventional ultrasound and ultrasonic elastography (UE) in preoperative diagnosis and combined diagnosis of malignant anus neoplasms was investigated. One hundred and twenty patients, whose mass was detected by digital rectal examination, were examined using UE and conventional ultrasound before operation, and the results were compared with those of histopathological examinations after operation, so that the accuracy and imaging features of UE as well as UE combined with conventional ultrasound in diagnosing malignant anus neoplasm were analyzed. Among the 120 patients, 77 were diagnosed with benign lesions and 43 were diagnosed with malignant lesions via pathological diagnosis for anal canal lesion surgery. Conventional ultrasound before operation showed that 53 patients were diagnosed with benign lesions and 26 with malignant lesions. Compared with that in the pathological diagnosis results, the diagnostic accordance rate in preoperative conventional ultrasound was $65.8 \%$. Through UE diagnosis, 66 patients had benign lesions and 39 patients had malignant lesions. Compared with that in the pathological diagnosis results, the diagnostic accordance rate in preoperative UE was $87.5 \%$. After the examination via UE combined with conventional ultrasound before operation, 71 patients were diagnosed with benign lesions and 40 patients were diagnosed with malignant lesions; compared with that in the pathological diagnosis results, the diagnostic accordance rate was $92.5 \%$. In terms of mass qualitative diagnosis, the sensitivity and specificity of conventional ultrasound were 60.5 and $68.8 \%$, respectively; those of UE were 90.7 and $85.7 \%$, respectively, and those of UE combined with conventional ultrasound were 93.0 and $92.2 \%$, respectively. According to the analysis results of receiver operating characteristic (ROC) curve, the area under curve (AUC) of malignant anus neoplasm diagnosed via UE was 0.732 [95\% confidence interval (95\% CI), 0.211-2.534], the AUC via conventional ultrasound was 0.695 (95\% CI, 0.517-
\end{abstract}

Correspondence to: Dr Quan Jiang, Department of Ultrasound, Shanghai Pudong New Area People's Hospital, 490 Chuanhuan South Road, Shanghai 201299, P.R. China

E-mail: shagw1@163.com

Key words: ultrasonic elastography, diagnosis, malignant anus neoplasm, receiver operating characteristic
0.932), and that via UE combined with conventional ultrasound was 0.823 (95\% CI, 0.146-4.643). In conclusion, examinations utilizing UE combined with conventional ultrasound can increase the preoperative diagnostic accordance rate in malignant anus neoplasm, which can be used as an effective method for preoperative diagnosis of malignant anus neoplasm.

\section{Introduction}

In China, malignant anus neoplasm is a relatively uncommon type of tumor (1), with incidence rate of $\sim 1.8-3.34 \%$ (2). Perianal cancer and rectal cancer are main types of malignant anus neoplasms occurring among males, while anal canal tumor is the main type of malignant anus neoplasms among females. With the increased incidence rate of tumors, the diagnosis of tumors becomes more and more important. Ultrasound $(3,4)$ is an essential method to assist the diagnosis of malignant anus neoplasm; therefore, ultrasound is frequently used for staging of malignant anus neoplasm in clinical practices. Ultrasonic diagnosis has many advantages, such as no trauma, high sensitivity of diagnosis, no radioactivity and simple and convenient methods; as a result, ultrasound has been widely applied in tumor diagnosis. Ultrasonic elastography (UE) (5) is an emerging tumor diagnosis technique, which expands the diagnostic scope of conventional ultrasound in tumors, can indicate and locate the positions of lesions and can differentiate the nature of lesions, having obvious advantages in judging the malignancy of tumors. In this study, some results were acquired from patients with anal canal masses who were diagnosed with conventional ultrasound combined with UE.

\section{Patients and methods}

Clinical data. One hundred and twenty patients, who were diagnosed in Shanghai Pudong New Area People's Hospital from December 2014 to December 2016, were selected; the anal canal masses were detected by digital rectal examination, then the nature of the masses was examined and determined using conventional ultrasound and UE, and all the patients were admitted in our hospital to receive operation. The study was approved by the Ethics Committee of Shanghai Pudong New Area People's Hospital and informed consents were signed by the patients or the guardians.

Apparatus and methods. Hi Vision Avius Color Doppler ultrasonic diagnostic apparatus (Hitachi,Ltd., Tokyo, Japan), equipped 
with a 13-5 probe, was used to perform UE and conventional ultrasound examinations. The positions of anal canal lesions, as well as the diameters, shape, edge conditions, calcification or not, thickness/length ratios of masses, in the patients were observed through ultrasound (6). After that, conventional ultrasound was switched to UE for examination. The three-grade classification, including benign, undetermined and malignant, was applied in conventional ultrasound. The UE scoring criteria provided by Hitachi, Ltd. were used as references for UE scoring $(7,8)$, of which UE score $\geq 3$ indicated malignant lesions.

Statistical analysis. All the experimental results were analyzed using SPSS 22.0 (IBM, Armonk, NY, USA), and analysis of variance was performed for comparisons among multiple groups. The enumeration data were expressed as percent (\%), and $\chi^{2}$ test was applied. The sensitivities and specificities of diagnoses via UE, conventional ultrasound and UE combined with conventional ultrasound in malignant anus neoplasm were calculated, respectively; the receiver operating characteristic (ROC) curves were used to investigate the diagnostic values of UE, conventional ultrasound and UE combined with conventional ultrasound in malignant anus neoplasm. $\mathrm{P}<0.05$ suggested that the difference was statistically significant.

\section{Results}

Basic data. There were 58 male patients and 62 female patients

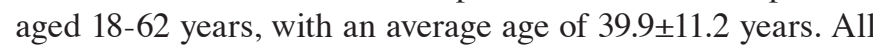
the postoperative masses of the 120 patients were diagnosed in the Department of Pathology. Results of pathological diagnosis: There were 43 cases of malignant anus neoplasm and 77 cases of benign anus neoplasm. The diameter of the mass was $1.2-3.5 \mathrm{~cm}$, with an average diameter of $1.6 \pm 0.8 \mathrm{~cm}$. Malignant melanoma was not included in this study (Table I).

Among the 120 patients, 77 were diagnosed with benign lesions and 43 were diagnosed with malignant lesions via pathological diagnosis for anal canal lesion surgery. Using conventional ultrasound before operation, 53 patients were diagnosed with benign anal canal masses and 26 with malignant anal canal masses; the total accordance rate was $65.8 \%$. Through UE diagnosis, 66 patients had benign anal canal masses and 39 patients had malignant anal canal masses, and the total accordance rate was $87.5 \%$. The numbers of patients diagnosed with benign and malignant masses via UE combined with conventional ultrasound were 77 and 43, respectively, and the total accordance rate was $92.5 \%$. Compared with the pathological diagnosis results, 24 cases of benign lesions were misdiagnosed as malignant ones, and 17 malignant lesions were misdiagnosed as benign ones in conventional ultrasound before operation. In preoperative UE, 11 cases of benign lesions were misdiagnosed as malignant ones, and 4 malignant lesions were misdiagnosed as benign ones. However, the diagnostic accuracy of UE combined with conventional ultrasound was relatively high (Table II).

Results of preoperative ultrasound examinations. Results of conventional ultrasound: Most of the benign masses were round or oval shape, with well-defined edges; typical microcalcification was not observed in the lesions; $88.4 \%$ (38/43) of the malignant lesions had irregular edges, $76.7 \%$ (33/43) of the masses had a thickness/length ratio $\geq 1$, and $62.8 \%(27 / 43)$ of
Table I. Clinical data of patients.

\begin{tabular}{lcc}
\hline Characteristics & Basic data & P-value \\
\hline Sex & & \\
Male & 58 cases & 0.347 \\
Female & 62 cases & \\
Age (years) & $39.9 \pm 11.2$ & \\
Nature of mass & & \\
Benign & 77 cases & 0.053 \\
Malignant & 43 cases & \\
Diameter of mass (cm) & $1.6 \pm 0.8$ & \\
Component of malignant & & \\
anus neoplasm & & \\
Cloacogenic carcinoma & 5 & \\
Basal cell carcinoma & 3 & \\
Epidermoid carcinoma & 15 & \\
or mucoepidermoid & & \\
carcinoma & & \\
Squamous cell carcinoma (SCC) & & \\
of anal canal & 20 & \\
\hline
\end{tabular}

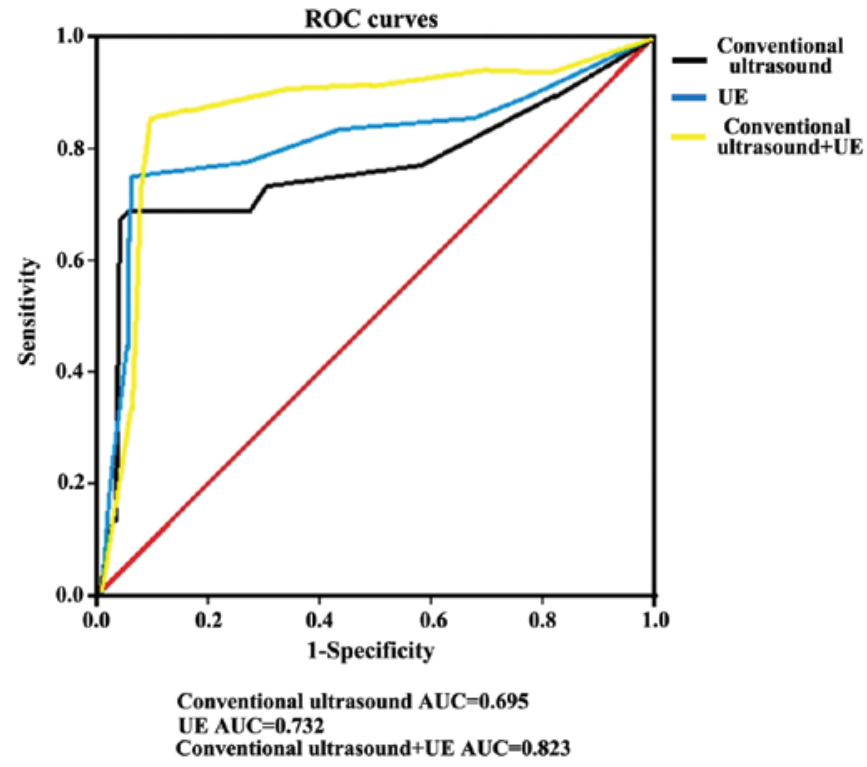

Figure 1. Receiver operating characteristic (ROC) curves of conventional ultrasound, ultrasonic elastography (UE) and UE combined with conventional ultrasound in diagnosing malignant anus neoplasm. Conventional ultrasound: $\mathrm{AUC}=0.695$; UE: $\mathrm{AUC}=0.732$; $\mathrm{UE}$ combined with conventional ultrasound: $\mathrm{AUC}=0.823$. The diagnostic value of $\mathrm{UE}$ combined with conventional ultrasound is relatively high.

the masses had micro-calcification. UE results: The elasticity score of the green region or the red-green region with green as the main color was 1-2 points, suggesting benign lesions, with surrounding tissues in green. The elasticity score of the red region was 3-5 points, suggesting malignant lesions, with surrounding tissues in red or green (Table III).

In terms of mass qualitative diagnosis, the sensitivity and specificity of conventional ultrasound were 60.5 and $68.8 \%$, 
Table II. Diagnostic results and accordance rates.

\begin{tabular}{|c|c|c|c|c|c|}
\hline Diagnostic results & & $\begin{array}{l}\text { Conventional } \\
\text { ultrasound }\end{array}$ & UE & $\begin{array}{c}\text { Conventional } \\
\text { ultrasound + EU }\end{array}$ & P-value \\
\hline \multirow[t]{2}{*}{ Benign lesion } & Detected & 53 & 66 & 71 & 0.372 \\
\hline & Misdiagnosed & 24 & 11 & 6 & \\
\hline \multirow[t]{2}{*}{ Malignant lesion } & Detected & 26 & 39 & 40 & 0.859 \\
\hline & Misdiagnosed & 17 & 4 & 3 & \\
\hline $\begin{array}{l}\text { Accordance rate of } \\
\text { pathological diagnosis }(\%)\end{array}$ & & 65.8 & 87.5 & 92.5 & 0.725 \\
\hline
\end{tabular}

UE, ultrasonic elastography.

Table III. Results of preoperative ultrasound examinations.

\begin{tabular}{lcc}
\hline Ultrasound feature & Conventional ultrasound & \\
\hline Benign lesion & Round and oval shape & Green: $1-2$ points \\
& Smooth edge & \\
& No typical micro-calcification & Red: 3-5 points \\
Thickness/length ratio $\geq 1$ & $88.4 \%$ & \\
Irregular edge & $76.7 \%$ & \\
Micro-calcification & $62.8 \%$ & \\
\hline
\end{tabular}

UE, ultrasonic elastography.

Table IV. Analyses of sensitivity and specificity of three diagnostic methods.

\begin{tabular}{lccc}
\hline Sensitivity and specificity & Conventional ultrasound & UE & Conventional ultrasound + EU \\
\hline Sensitivity (\%) & 60.5 & 90.7 & 93.0 \\
Specificity (\%) & 68.8 & 85.7 & 92.2 \\
AUC & 0.695 & 0.732 & 0.823 \\
$95 \%$ CI & $0.517-0.932$ & $0.211-2.534$ & $0.146-4.643$ \\
\hline
\end{tabular}

UE, ultrasonic elastography; AUC, area under curve.

respectively; those of UE were 90.7 and $85.7 \%$, respectively, and those of UE combined with conventional ultrasound were 93.0 and $92.2 \%$, respectively. According to the analysis results of ROC curve, the area under curve (AUC) of malignant anus neoplasm diagnosed via UE was 0.732 [95\% confidence interval (95\% CI), 0.211-2.534], the AUC via conventional ultrasound was 0.695 (95\% CI, 0.517-0.932), and that via UE combined with conventional ultrasound was 0.823 (95\% CI, 0.146-4.643) (Table IV and Fig. 1).

\section{Discussion}

Malignant anus neoplasm (9) is an uncommon type of cancer, but its incidence rate shows an increasing trend, and the patients with the disease become increasingly younger (10). Therefore, the screening of malignant anus neoplasm is becoming more important. Based on ultrasonic diagnosis, UE (11) conducts further examinations on the lesions. The detection principles of
UE (12) are as follows: The mass to be detected is compressed toward the probe, then the distributions of elastic coefficient and strain of the detected mass are calculated according to its displacement, and the figures for the results are formulated. Conventional ultrasound combined with UE can increase the diagnostic accuracy in malignant anus neoplasm.

In this study, the detection results of conventional ultrasound in malignant anus neoplasm showed unclear edges, irregular shapes and increased anterior-posterior diameter. On determining whether the tumor is malignant or not, thickness/length ratio $>1$ is a crucial index (13). The study results revealed that there were 13 cases of lesions which were diagnosed as undetermined via conventional ultrasound but were determined as benign via UE; 14 cases had a score $>3$ points in UE, which were judged as benign lesions through conventional ultrasound. After the examinations by UE combined with conventional ultrasound, the sensitivity and accuracy of the diagnosis of malignant anus neoplasm were improved significantly, thus avoiding missed diagnosis 
and misdiagnosis. Currently, there are plenty of studies on the diagnosis of breast cancer via UE (14-18); UE has good values in the diagnosis of breast cancer, its sensitivity and accuracy are relatively high; there is no great difference in diagnosis of the malignancy of breast lesion between 5-point scoring evaluation and UE area ratio, while the diagnosis combined with the two methods can increase the diagnostic accuracy of tumors. UE has not been completely utilized as a routine examination for tumors in clinic yet, and there are no unified diagnostic criteria. Some studies revealed (19-21) that the malignancy of breast lesion may cause adverse reactions to the UE diagnostic results, and the accuracy of UE examination is decreased along with the increased depth of the mass. It was indicated in the ROC curve that UE had significant values in diagnosis of malignant anus neoplasm, and that the diagnostic value of UE combined with conventional ultrasound was greater. Some studies have revealed that UE has great values in diagnosis of liver fibrosis (22). In the examination of masses, on the basis of lesion detection via conventional ultrasound, lesions that cannot be confirmed by conventional ultrasound are discovered and diagnosed using UE examination, and the prognostic value in the lesions needs to be further investigated.

In this study, the diagnostic effects of UE and conventional ultrasound in malignant anus neoplasm were studied, and the results showed that 4 cased were diagnosed as suspected malignant masses via UE, with a score $\geq 3$ points, which were diagnosed as benign ones through pathological diagnosis. There was a relatively high misdiagnosis rate of malignant lesions using UE alone in this study. It was considered that misdiagnosis may be associated with many reasons, such as depth of mass and too few points of interest during the diagnosis. Since there are no reports related to the diagnosis of UE and conventional ultrasound in malignant anus neoplasm, a small sample size is adopted in this study, which lacks representativeness; therefore, the results need to be verified by large quantity of samples and data findings.

In conclusion, in the clinical diagnosis of malignant anus neoplasm at present, examinations through conventional ultrasound combined with UE has a high diagnostic accuracy, and the devices used during popularization are relatively simple; therefore, conventional ultrasound combined with UE can be used as an effective method for preoperative diagnosis of malignant anus neoplasm.

\section{Acknowledgements}

This study is supported by Pu Dong New Area Health and Family Planning Commission Important Vulnerable Course Project (no. PWzbr 2017-10) and Pu Dong New Area Health and Family Planning Commission Subject leader Course Project (no. PWRd 2017-06).

\section{References}

1. Morris V, Rao X, Pickering C, Foo WC, Rashid A, Eterovic K, Kim T, Chen K, Wang J, Shaw K, et al: Comprehensive genomic profiling of metastatic squamous cell carcinoma of the anal canal. Mol Cancer Res 15: 1542-1550, 2017.

2. Zimmermann M, Beer J, Bodis S, von Moos R, Vlachopoulou V, Zwahlen DR and Oehler C: PET-CT guided SIB-IMRT combined with concurrent 5-FU/MMC for the treatment of anal cancer. Acta Oncol 30: 1-7, 2017.
3. Zhang XL and Qian LX: Ultrasonic features of papillary thyroid microcarcinoma and non-microcarcinoma. Exp Ther Med 8: 1335-1339, 2014.

4. Li B, Zhang Y, Yin P, Zhou J and Jiang T: Ultrasonic features of papillary thyroid microcarcinoma coexisting with a thyroid abnormality. Oncol Lett 12: 2451-2456, 2016.

5. Lu R and Xiao Y: The diagnostic value of ultrasonic elastography and ultrasonography comprehensive score in cervical lesions. Zhonghua Yi Xue Za Zhi 97: 2111-2115, 2017.

6. Tyloch JF and Wieczorek AP: The standards of an ultrasound examination of the prostate gland. Part 2. J Ultrason 17: 43-58, 2017.

7. Hahn S, Lee YH, Lee SH and Suh JS: Value of the strain ratio on ultrasonic elastography for differentiation of benign and malignant soft tissue tumors. J Ultrasound Med 36: 121-127, 2017.

8. Yağc1 B, Erdem Toslak I, Çekiç B, Öz M, Karakaş BR, Akdemir M, Yildız S, Süren D and Bova D: Differentiation between idiopathic granulomatous mastitis and malignant breast lesions using strain ratio on ultrasonic elastography. Diagn Interv Imaging 98: 685-691, 2017.

9. Torkzad MR, Kamel I, Halappa VG and Beets-Tan RG: Magnetic resonance imaging of rectal and anal cancer. Magn Reson Imaging Clin N Am 22: 85-112, 2014.

10. Rödel F, Wieland U, Fraunholz I, Kitz J, Rave-Fränk M, Wolff HA, Weiss C, Wirtz R, Balermpas P, Fokas E, et al: Human papillomavirus DNA load and p16INK4a expression predict for local control in patients with anal squamous cell carcinoma treated with chemoradiotherapy. Int J Cancer 136: 278-288, 2015.

11. Li LJ, Zeng H, Ou B, Luo BM, Xiao XY, Zhong WJ, Zhao XB, Zhao ZZ, Yang HY and Zhi H: Ultrasonic elastography features of phyllodes tumors of the breast: A clinical research. PLoS One 9: e85257, 2014

12. Mu WJ, Zhong WJ, Yao JY, Li LJ, Peng YL, Wang Y, Liu LS, Xiao Y, Liu SJ, Wu CJ, et al: Ultrasonic elastography research based on a multicenter study: Adding strain ratio after 5-point scoring evaluation or not. PLoS One 11: e0148330, 2016.

13. Mainiero MB, Lourenco A, Mahoney MC, Newell MS, Bailey L, Barke LD, D'Orsi C, Harvey JA, Hayes MK, Huynh PT, et al: ACR appropriateness criteria breast cancer screening. J Am Coll Radiol 10: 11-14, 2013.

14. Ahmed M, Jozsa F, Baker R, Rubio IT, Benson J and Douek M: Meta-analysis of tumour burden in pre-operative axillary ultrasound positive and negative breast cancer patients. Breast Cancer Res Treat 10: 1-8, 2017.

15. Diepstraten SC, Sever AR, Buckens CF, Veldhuis WB, van Dalen T, van den Bosch MA, Mali WP and Verkooijen HM: Value of preoperative ultrasound-guided axillary lymph node biopsy for preventing completion axillary lymph node dissection in breast cancer: A systematic review and meta-analysis. Ann Surg Oncol 21: 51-59, 2014.

16. Berg WA, Bandos AI, Mendelson EB, Lehrer D, Jong RA and Pisano ED: Ultrasound as the primary screening test for breast cancer: Analysis from ACRIN 6666. J Natl Cancer Inst 108: 367, 2015.

17. Qi X, Chen A, Zhang P, Zhang W, Cao X and Xiao C: Mammographic calcification can predict outcome in women with breast cancer treated with breast-conserving surgery. Oncol Lett 14: 79-88, 2017.

18. Wu X, Lin Q, Lu J, Chen G, Zeng YI, Lin Y, Chen Y, Wang Y and Yan J: Comparison of mammography and ultrasound in detecting residual disease following bioptic lumpectomy in breast cancer patients. Mol Clin Oncol 4: 419-424, 2016.

19. Evans A, Rauchhaus P, Whelehan P, Thomson K, Purdie CA, Jordan LB, Michie CO, Thompson A and Vinnicombe S: Does shear wave ultrasound independently predict axillary lymph node metastasis in women with invasive breast cancer? Breast Cancer Res Treat 143: 153-157, 2014.

20. Mi XK, Liu QR, Zhu L, Sang MX, Guo LR and Shan BE: Mechanism of the high coagulation state of breast cancer tissue factor. Eur Rev Med Pharmacol Sci 21: 2167-2171, 2017.

21. Ganott MA, Zuley ML, Abrams GS, Lu AH, Kelly AE, Sumkin JH, Chivukula M, Carter G, Austin RM and Bandos AI: Ultrasound guided core biopsy versus fine needle aspiration for evaluation of axillary lymphadenopathy in patients with breast cancer. ISRN Oncol 2014: 703160, 2014.

22. Qi JS, Wang WH and Li FQ: Combination of interventional adenovirus-p53 introduction and ultrasonic irradiation in the treatment of liver cancer. Oncol Lett 9: 1297-1302, 2015.

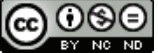

This work is licensed under a Creative Commons Attribution-NonCommercial-NoDerivatives 4.0 International (CC BY-NC-ND 4.0) License. 